\title{
Reliability of a standing isokinetic shoulder rotators strength test using a functional electromechanical dynamometer: Effects of velocity
}

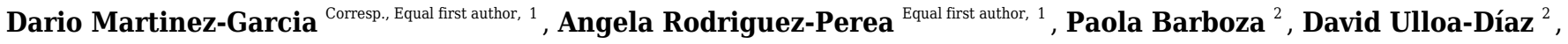 \\ Daniel Jerez-Mayorga ${ }^{3}$, Ignacio Chirosa ${ }^{1}$, Luis Javier Chirosa Ríos ${ }^{1}$ \\ 1 Department of Physical Education, Universidad de Granada, Granada, Andalucia, Spain \\ 2 Department of Sports Sciences and Physical Conditioning, Catholic University of Most Holy Concepcion, Concepción, Bio Bio, Chile \\ 3 Faculty of Rehabilitation Sciences, Universidad Andres Bello, Santiago, Santiago, Chile \\ Corresponding Author: Dario Martinez-Garcia \\ Email address: dariomg@correo.ugr.es
}

Background. The evaluation of the force in internal rotation (IR) and external rotation (ER) of the shoulder is commonly used to diagnose possible pathologies or disorders in the glenohumeral joint and to assess patient's status and progression over time. Currently, there is new technology of multiple joint isokinetic dynamometry that allows to evaluate the strength in the human being. The main purpose of this study was to determine the absolute and relative reliability of concentric and eccentric internal and external shoulder rotators with a functional electromechanical dynamometer (FEMD).

Methods. Thirty-two male individuals ( $21.46 \pm 2.1$ years) were examined of concentric and eccentric strength of shoulder internal and external rotation with a FEMD at velocities of $0.3 \mathrm{~m} \cdot \mathrm{s}-1$ and $0.6 \mathrm{~m} \cdot \mathrm{s}-1$. Relative reliability was determined by intraclass correlation coefficients (ICC). Absolute reliability was quantified by standard error of measurement (SEM) and coefficient of variation (CV). Systematic differences across velocities testing circumstances, were analyzed with dependent $t$ tests or repeatedmeasures analysis of variance in case of 2 or more than 2 conditions, respectively.

Results. Reliability was high to excellent for IR and ER on concentric and eccentric strength measurements, regardless of velocity used (ICC: 0.81-0.98, CV:5.12-8.27\% SEM: 4.06-15.04N). Concentric outcomes were more reliable than eccentric due to the possible familiarization of the population with the different stimuli.

Conclusion. All procedures examined showed high to excellent reliability for clinical use. However, a velocity of $0.60 \mathrm{~m} \cdot \mathrm{s}-1$ should be recommended for asymptomatic male patients because it demands less time for evaluation and patients find it more comfortable. 
1 Reliability of a standing isokinetic shoulder rotators

2 strength test using a functional electromechanical

3 dynamometer: Effects of velocity

Dario Martinez-Garcia ${ }^{1}$, Angela Rodriguez-Perea ${ }^{1}$, Paola Barboza-Gonzalez ${ }^{2}$, David Ulloa-

Diaz $^{2}$, Daniel Jerez-Mayorga ${ }^{4}$, Ignacio Chirosa ${ }^{1}$, Luis Javier Chirosa Rios ${ }^{1}$.

7

8

${ }^{1}$ Department of Physical Education, Universidad de Granada, Granada, Spain

${ }^{2}$ Department of Sports Sciences and Physical Conditioning, Catholic University of Most Holy Concepcion, Concepción, Bio Bio, Chile

${ }^{3}$ Faculty of Rehabilitation Sciences, Universidad Andres Bello, Santiago, Chile

Corresponding Author:

\section{Dario Martinez-Garcia ${ }^{1}$}

Camino de Alfacar s/n, Granada, Granada, 18011, España

Email address: dariomg@correo.ugr.com

\section{Abstract}

Background. The evaluation of the strength in internal rotation (IR) and external rotation (ER) of the shoulder is commonly used to diagnose possible pathologies or disorders in the glenohumeral joint and to assess patient's status and progression over time. Currently, there is a new technology of multiple joint isokinetic dynamometry that allows us to evaluate the strength in the human being. The main purpose of this study was to determine the absolute and relative reliability of concentric and eccentric internal and external strength shoulder rotators with a functional electromechanical dynamometer (FEMD).

Methods. Thirty-two male individuals $(21.46 \pm 2.1$ years $)$ were examined of concentric and eccentric strength of shoulder internal and external rotation with a FEMD at velocities of 0.3 $\mathrm{m} \cdot \mathrm{s}^{-1}$ and $0.6 \mathrm{~m} \cdot \mathrm{s}^{-1}$. Relative reliability was determined by intraclass correlation coefficients (ICC). Absolute reliability was quantified by the standard error of measurement (SEM) and coefficient of variation (CV). Systematic differences across velocities testing circumstances were analyzed with dependent t-tests or repeated-measures analysis of variance in case of 2 or more than two conditions, respectively.

Results. Reliability was high to excellent for IR and ER on concentric and eccentric strength measurements, regardless of velocity used (ICC: 0.81-0.98, CV:5.12-8.27\% SEM: 4.06-15.04N). Concentric outcomes were more reliable than eccentric due to the possible familiarization of the population with different stimuli. 
35 Conclusion. All procedures examined showed high to excellent reliability for clinical use.

36

37

38

39

40

41

42

43

44

45

46

47

48

49

50

51

52

53

54

55

56

57

58

59

60

61

62

63

64

65

66

67

68

69

70

71

72
However, a velocity of $0.60 \mathrm{~m} \cdot \mathrm{s}^{-1}$ should be recommended for asymptomatic male patients because it demands less time for evaluation

Keywords. isokinetic, shoulder, strength, reproducibility, position, velocity.

\section{Introduction}

Clinicians and researchers periodically assess strength changes in the status of their patients (McLaine, Ginn, Kitic, Fell, \& Bird, 2016). The evaluation of the strength in internal rotation (IR) and external rotation (ER) of the shoulder is commonly used to (I) diagnose possible pathologies or disorders in the glenohumeral joint, (II) evaluate the effectiveness and progression of the proposed treatment, and (III) as well as to quantify the change in muscle quality and the development of strength over time (Cools et al., 2014; Edouard et al., 2011). Also, objective measurements of this variable have proved to be essential for the identification of a patient at risk of injury, especially in athletes, against subjective measures or the self-report outcome score (Cools, Johansson, Borms and Maenhout, 2015). Clinicians and researchers should have accurate and reliable protocol tests that objectively assess changes in strength over time that reflect the real gain or loss due to the protocol measurement (Edouard et al., 2011; Johansson et al., 2015).

The reliability measures in test-retest concern the repeatability of the data observed in a population in repeated measures (Hopkins, 2000). In medicine, it is a requirement to have relative reliability data with intraclass correlation coefficients (ICCs) and absolute reliability with a standard error of measurement (SEM) and coefficient of variation (CV) (Hopkins, Schabort, \& Hawley, 2001). The relative reliability indicates how well the rank order of the participants in the test are similar to the retest. The main problem with relative reliability is that it depends on the variability of the sample (Hopkins, 2000; Hopkins et al., 2001). However, absolute reliability is related to the consistency of individual scores (Hopkins et al., 2001; Weir, 2005).

Multiple tests have been created for the evaluation of shoulder strength with a wide variety of tools, including manual muscle testing (García, 2006; Lu, Hsu, Chang, \& Chen, 2007), handheld dynamometers (Chamorro, Armijo-Olivo, De La Fuente, Fuentes, \& Chirosa, 2017; Cools et al., 2014; Holt, Raper, Boettcher, Waddington, \& Drew, 2016) or isokinetic devices (Andrade, Fleury, de Lira, Dubas, \& da Silva, 2010; Radaelli, Bottaro, Weber, Brown, \& Pinto, 2010; Stickley, Hetzler, Freemyer, \& Kimura, 2008). Isokinetic devices are considered as the gold standard in strength evaluations since they allow you to evaluate the maximum dynamic force throughout the range of motion (ROM) (Caruso, Brown, \& Tufano, 2012). Due to the biomechanics of the shoulder joint and its wide mobility, the reliability of the measurements in isokinetic devices are influenced by many factors (mechanical aspects, subjects, joints, and testing protocols), the assessment position, including the position of the shoulder (shoulder 
73

74

75

76

77

78

79

80

81

82

83

84

85

86

87

88

89

90

91

92

93

94

95

96

97

98

99

100

101

102

103

104

105

106

107

108

109

posture and joint-axis alignment) and the position of the body (sitting, supine, or standing and stabilization)(Broztman \& Wilk, 2005; Edouard et al., 2011). Also, there are discrepancies in the literature regarding the most reliable velocities for testing. Additionally, most previous studies investigated the reproducibility of shoulder measurements between $60 \% \mathrm{~s}$ and $240 \% \mathrm{~s}$ (Nugent, Snodgrass, \& Callister, 2015). Higher velocities seem to be important when power assessment is necessary (the ability to produce moment rapidly) but less reliable. Some authors report faster velocities to be more reliable, while others report that low speeds are more reliable for measuring shoulder rotator's strength (Edouard et al., 2011; Nugent et al., 2015).

Nowadays, a new multiple joint isokinetic dynamometry has appeared (Dvir \& Müller, 2019), denominated functional electromechanical dynamometry (FEMD). It allows us to evaluate the strength in the human being. The isometric strength of the shoulder rotators (Chamorro, De La Fuente, Jerez, Campos, \& Chirosa, 2018) and hip abductor strength (Cerda Vega et al., 2018) has been studied with this technology; however, shoulder maximal dynamic strength has not been studied yet (Claudio Chamorro et al., 2018) (Figure 1).

\section{*Figure 1 here*}

FEMD provides a quantified measurement of strength, and it is easy to use and has a lower cost compared to other isokinetic devices. Unlike others, this machine generates linear isokinetic speeds, dynamic modes (tonic, kinetic, elastic, inertial, conical) and statics modes (isometric, vibratory), allowing the evaluation and training through constant and/or variable resistance/velocity (Campos Jara et al., 2014). Furthermore, it has been described as a valid and reliable measurement method for evaluating upper and lower extremity muscle strength (Cerda Vega et al., 2018; Chamorro et al., 2018). This technology has been used to study the isometric strength of the shoulder rotators and has obtained high-reliability values (ICC > 0.94; CV < 10\%) (Chamorro et al., 2018; Chamorro, de la Fuente, Rubio, Campos, \& Chirosa, 2019) In addition, this device has also been used to evaluate other joints such as lower limbs (Chamorro et al., 2017; Fàbrica, Ferraro, Mercado-Palomino, Molina-Molina, \& Chirosa-Rios, 2020) and trunk (Rodriguez-Perea et al., 2019).

Therefore, the main purpose of this study was (I) to determine the absolute and relative reliability of concentric and eccentric internal and external shoulder rotators in standing position with FEMD in the evaluation of the isokinetic strength to determine the most reliable test condition, and (II) To compare the absolute and relative reliability of different velocities for the assessment of isokinetic test. We hypothesized that (I) this new test will be a reliable method for the assessment of concentric and eccentric strength in shoulder rotators low speed would be more reliable than high speeds. The results are expected to provide new information regarding the dynamic shoulder strength evaluation protocols with FEMD.

\section{Materials \& Methods}


110

111

112

113

114

115

116

117

118

119

120

121

122

123

124

125

126

127

128

129

130

131

132

133

134

135

136

137

138

139

140

141

142

143

144

145

146

\section{Participants}

Thirty-two asymptomatic male volunteers were recruited from local university (age: $21.46 \pm 2.1$ years, body mass: $69.22 \pm 6.85 \mathrm{~kg}$, height: $1.73 .5 \pm 0.07 \mathrm{~m}$, Shoulder Pain and Disability Index (SPADI): $15.2 \pm 3.8$ and body mass index (BMI): $22.98 \pm 1.607 \mathrm{~kg} / \mathrm{m}^{2}$ ) without any experience in isokinetic or dynamometer devices participated in this study. Participants were eligible for the study if they were: i) free of shoulder pain, with a maximum of $20 \%$ Shoulder Pain and Disability Index (SPADI); ii) free of musculoskeletal injury; iii) not practice specific training of upper body strength; and iv) a maximum BMI of $25 \mathrm{~kg} / \mathrm{m}^{2}$. All participants were informed regarding the nature, aims, and risks associated with the experimental procedure before they gave their written consent to participate. The study protocol was approved by the Biomedical Committee of the University $\left(n^{\circ} 350 / \mathrm{CEIH} / 2017\right)$ and was conducted in accordance with the Helsinki Declaration.

\section{Study design}

A repeated-measurement design was used to evaluate the shoulder rotator strength with different protocols. After two familiarization sessions, participants attended the laboratory on four separate days (at least 48 hours apart) for two weeks. On each testing, day participants completed two sessions of familiarization and two protocol sessions with $0.3 \mathrm{~m} \cdot \mathrm{s}^{-1}$ or $0.6 \mathrm{~m} \cdot \mathrm{s}^{-1}$. Participants were asked to maintain their physical activity level during the two weeks of the study. All evaluations were conducted at the same time of the day $( \pm 1 \mathrm{~h})$ for each participant and under similar environmental conditions $\left(\sim 21^{\circ} \mathrm{C}\right.$ and $\sim 60 \%$ humidity $)$. The order of velocities was randomly established.

\section{Instrument}

Isometric and isokinetic strength were evaluated with a FEMD (DynaSystem, Model Research, Granada, Spain) with a precision of three $\mathrm{mm}$ for displacement, $100 \mathrm{~g}$ for a sensed load, a sampling frequency of $1,000 \mathrm{~Hz}$ and a range of velocities between $0.05 \mathrm{~m} \cdot \mathrm{s}^{-1}$ to $2.80 \mathrm{~m} \cdot \mathrm{s}^{-1}$. Subjects can perform a wide variety of movements, and the device can deliver a wide variety of stimuli (isokinetic, isotonic, elastic, isometric, inertial, eccentric, and vibration). Its control core precisely regulates both force and angular velocity through a $2000 \mathrm{~W}$ electric motor. The user applies forces on a rope that winds on a roller, thus controlling and measuring both force and linear velocity. A load cell detects the tension applied to the rope, and the resulting signal goes to an analog-to-digital converter with 12-bit resolution. Displacement and speed data are collected with a 2500 ppr encoder attached to the roller. Data from the different sensors are obtained at a frequency of $1 \mathrm{kHz}$ (Fig 2).

\section{*Figure 2 here*}

Range of movement

Subjects were positioned standing and supporting the dominant upper limb on a subjection system of own manufacture (Fig 3). The subjection system was regulated, taking into account the 
147 subject's height with a variation of $\pm 1 \mathrm{~cm}$. The humerus was fixed with a cinch at $2 / 3$ of the 148 distance between the lateral epicondyle and the acromion. Both the position and the range of 149 movement of the same was determined with a baseline goniometer (Gymna hoofdzetel, Bilzen, 150 Belgium). The position consisted of a $90^{\circ}$ adduction of the glenohumeral joint and a $90^{\circ}$ flexion

151

152

153

154

155

156

157

158

159

160

161

162

163

164

165

166

167

168

169

170

171

172

173

174

175

176

177

178

179

180

181

182

183

184

185 of the humero-ulnar joint. For the glenohumeral joint, the fulcrum was positioned in the acromion with the vertical arm stable and the arm movable along the humerus with the lateral epicondyle as a point of reference. For the humero-ulnar articulation, the fulcrum was positioned in the lateral epicondyle with the arm stable in horizontal and the arm movable along the forearm with the processus styloideus ulnae as a reference point. The range of movement was measured during the two familiarization sessions. The fulcrum is positioned in the olecranon with the stable arm in vertical (IR) or horizontal (ER) position and the mobile arm along the forearm with the processus styloideus ulnae as a reference until the goniometer is at an angle of $90^{\circ}$ registering this distance to keep it stable throughout the different measurements.

\section{*Figure 3 here*}

\section{Familiarization Protocol}

Participants first attend (four subjects each time) to 90 minutes familiarization sessions with the FEMD and to testing the procedures. The familiarization consisted of a general warm-up for both test session consisted on 5 min of jogging (beats per minute $<130$; measurement with a Polar M400), 5 min of joint mobility and 1 set of 5 repetitions of shoulder flexion-extension, five repetitions abduction and shoulder adduction, and five repetitions of IR and ER in the position of the test that was to be performed. After this, the same evaluation protocols that were going to be carried out in the evaluations were carried out.

\section{Test Protocol}

Participants arrived in a well-rested condition at the start of each testing session. After the same warm-up of familiarization protocol, participants rested for $5 \mathrm{~min}$ before the initiation. The test consisted of two series of 5 maximum consecutive repetitions of shoulder rotators at a velocity of $0.60 \mathrm{~m} \cdot \mathrm{s}^{-1}$ and $0.30 \mathrm{~m} \cdot \mathrm{s}^{-1}$ and with the range of movement previously established. The rest between sets was a three-minute. The three highest repetitions of the mean force for the concentric contraction and the eccentric contraction were taken to calculate the mean dynamic force for each participant and velocity. The average strength of the total repetitions was taken into account in this measurement (Figure 2).

\section{Statistical Analysis}

The descriptive data are presented as mean $\pm \mathrm{SD}$. The distribution of the data was verified for the first time by the Shapiro-Wilk normality test. Reliability was assessed by t-tests of paired samples with the effect size ES, CV, and ICC, with $95 \%$ confidence intervals. The scale used for interpreting the magnitude of the ES was: negligible $(<0.2)$, small $(0.2-0.5)$, moderate $(0.5-0.8)$, and large $(\geq 0.8)$ (Cohen, 1988). Following Hopkins et al. (2009) classify through a qualitative scale the magnitude of the values of the ICC, being the values close to 0.1 low reliability, 0.3 
186

187

188

189

190

191

192

193

194

195

196

197

198

199

200

201

202

203

204

205

206

207

208

209

210

211

212

213

214

215

216

217

218

219

220

221

222

moderate, 0.5 high, 0.7 very high, and those close to 0.9 extremely high. Sensitivity was estimated by the smallest detectable change (SDC) derived from the SEM (Courel-Ibáñez et al., 2019). The level of agreement between paired velocity outcomes from two measures was also assessed using Bland-Altman plots and the calculation of systematic bias and its $95 \%$ limits of agreement $(\mathrm{LoA}=$ bias $\pm 1.96 \mathrm{SD})$. Maximum errors (Max Error) at the 95\% confidence interval were calculated from the Bland-Altman bias (Max Error) for the different condition outcomes analyzed (Paalanne et al., 2009). Reliability analyzes were performed using a customized spreadsheet (Hopkins et al., 2009) while the JASP software package (version 0.9.1.0, http://www.jasp-stats.org) was used for all other analyses. Figures were designed using GraphPad Prism 6.0 (GraphPad Software Inc., California, USA).

\section{Results}

Relative reliability for internal rotation test had high or excellent in all conditions (ICC: $0.81-$ 0.93). Absolute reliability ranged from $6.31 \%$ to $8.27 \% ; 5.58 \mathrm{~N}$ to $6.8 \mathrm{~N}$ in concentric contractions and $14.98 \mathrm{~N}$ to $15.04 \mathrm{~N}$ in eccentric contractions for CV and SEM respectively. ES was negligible in any of the conditions for internal rotation test $(0.00-0.16)$ (Table 1$)$.

\section{*Table 1 near here*}

For external rotation tests, relative reliability test had high or excellent in all conditions (ICC: $0.89-0.98)$. Absolute reliability ranged from $5.12 \%$ to $6.91 \% ; 4.06 \mathrm{~N}$ to $4.19 \mathrm{~N}$ in concentric contractions and $10.16 \mathrm{~N}$ to $13.64 \mathrm{~N}$ in eccentric contractions for CV and SEM respectively. ES was negligible in any of the conditions for external rotation test $(-0.19-0.10)$, except for a small ES in concentric contraction in very slow velocity (ES: 0.24) (Table 1)

\section{*Figure 4 here*}

Bland-Altman plots showed the highest agreement and the most regular variation but exhibited more variation in eccentric contractions. Analysis of systematic biases by paired student $\mathrm{t}$-tests found no significant difference except for external rotation in concentric contraction at $0.3 \mathrm{~m} \cdot \mathrm{s}^{-1}$ and eccentric contraction at $0.6 \mathrm{~m} \cdot \mathrm{s}^{-1}$ (Figures 4 and 5).

\section{*Figure 5 here*}

\section{Discussion}

The main purpose of this study was to determine the absolute and relative reliability of concentric and eccentric internal and external shoulder rotators in a standing position with a FEMD. To our knowledge, this was the first study to assess reliability for dynamic shoulder strength tests performed on a FEMD. The present investigation shows high to excellent reliability with ICC and CV values ranging from 0.81 to 0.93 and $6.31-8.27 \%$ for IR and from 0.89 to 0.98 and $5.12-6.91 \%$ for ER. Our findings confirm that this standing test is a reliable method for assessing shoulder rotation strength in asymptomatic adults. These results are of clinical relevance because shoulder rotation strength may be a useful marker of shoulder joint function. 
223 Patients' position in the evaluations of shoulder strength is a relevant variable which has to take

224

225

226

227

228

229

230

231

232

233

234

235

236

237

238

239

240

241

242

243

244

245

246

247

248

249

250

251

252

253

254

255

256

257

258

259

260

261 into account for the reliability of the outcome of the test, due to the complex structure of the glenohumeral joint (Edouard et al., 2011; Forthomme, Dvir, Crielaard, \& Croisier, 2011). There is no scientific consensus about which the best position is to obtain reliable strength data in the shoulder test. The three positions most used by clinicians are supine (Andersen, Vinstrup, Jakobsen, \& Sundstrup, 2017; Claudio Chamorro et al., 2018; Forthomme et al., 2011), sitting (Byram et al., 2010; Edouard et al., 2013; Radaelli et al., 2010) and standing (Frisiello, Gazaille, O’Halloran, Palmer, \& Waugh, 1994; Greenfield, Donatelli, Wooden, \& Wilkes, 1990). The sitting position has been the most widely studied due to the clinical nature of the studies and to an easier stabilization of the patient when evaluating (Edouard et al., 2011). Whether they are asymptomatic patients, athletes, or patients with chronic pain, the daily movements where the glenohumeral joint is used are usually not in this position (Beneka, Mailliou, Giannakopoulos, Kyrialanis, \& Godolias, 2002; Rodríguez-Rosell, Pareja-Blanco, Aagaard, \& González-Badillo, 2017). Therefore, in this study ${ }_{2}$ we propose the creation of a standing test, closer to the actual position of patients in the movements that encompass the shoulder. The results of this study show that standing tests can be performed reliably for asymptomatic patients. This study has obtained similar or better relative and absolute reliability data (ICC: 0.81 to $0.98, \mathrm{CV}: 5.12$ to 8.27) than most studies with this evaluation position Greenfield et al. (1990) (ICC: $0.81-0.95$ ) and Frisiello et al. (1994) (ICC: 0.77 - 0.86) and similar data to articles with a sitting position for the same type of population: Mayer, Horstmann, Kranenberg, Röcker, \& Dickhuth, 1994 (ICC: 0.09 - 0.89; SEM: 2.8 - 9.2), Kramer \& Ng, 1996 (ICC: 0.83 - 0.96; SEM: 2 - 7) or Malerba, Adam, Harris, \& Krebs, 1993 (ICC: $0.44-0.90$ ).

Second aim of the study was to compare the absolute and relative reliability of different velocities for the assessment of the isokinetic test. Velocity in isokinetic tests has been a widely studied variable in the literature (Andrade et al., 2010; Hadzic, Ursej, Kalc, \& Dervisevic, 2012; Zanca, Oliveira, Saccol, \& Mattiello-Rosa, 2011). Although, most studies agree that speeds below $60 \%$ s are usually the most reliable to perform strength assessments in most joints (Nugent et al., 2015). There is no widely accepted consensus on which of these speeds is best suited for the shoulder joint (Edouard et al., 2011). In our study, we compared two speeds, one slow and one very slow to see if, as the velocity are lower, the reliability of the isokinetic test increased or, on the contrary, controlled slow speeds, but closer to the reality of the daily gestures of the patients produced a more reproducible application of force in these tests. Our results agree with this second idea, as previously noted in (Castro et al., 2017; Hadzic et al., 2012). While both velocities are reliable, the slow speed presents better results compared to the very slow one, as we can see in the Bland-Altman plots (Figure 4 and 5), given that their bias are closer to 0 and most points are within the limits of agreement (LoA).

However, our analysis revealed large differences between the concentric and eccentric phases of both IR and ER. One possible explanation is that eccentric isokinetic tests require greater familiarization in IR while it was more reliable in the ER (Hadzic et al., 2012). 
262 Bland-Altman bias (Max Error) for the different condition outcomes found no significant 263 difference except for external rotation in concentric contraction at $0.3 \mathrm{~m} \cdot \mathrm{s}^{-1}$ and eccentric

264

265

266

267

268

269

270

271

272

273

274

275

276

277

278

\section{9}

280

281

282

283

284

285

286

287

288

289

290

291

292

293

294

295

296

297

298

299

300

301

contraction at $0.6 \mathrm{~m} \cdot \mathrm{s}^{-1}$. This may be due to natural movements of the shoulder in the population tested, where the ER phase is predominantly eccentric, while the IR contraction is substantially concentric (Wagner et al., 2012). However, limits of agreement are wider in an eccentric phase like results obtained in (Pallarés et al., 2014), testing a stop between phases for a better adaptation of the subjects (Figures 4 and 5). Clinicians and trainers should take these results into account to individualize the evaluation protocols according to the type of population and problem, whether they are overhead athletes or populations at risk of chronic pain in the shoulder.

Some limitations of this study should be noted to take them into account when evaluating our results. It was not possible to perform an inter-rater reliability analysis, so we can not know how this variable affects evaluations. We recommend both clinicians and coaches always have the same evaluator with the same patient. Also, the study was performed with asymptomatic active patients, which means that the results can not be extrapolated to other types of the population such as the sedentary population, or patients with chronic pain in the shoulder. Further study of these variables would be necessary to standardize the results to any type of population.

\section{Conclusion}

The study results show high to excellent reliability values for all procedures performed. Based on the results of this study, it may be reasonably concluded that the assessment in a standing position either at $0.6 \mathrm{~m} \cdot \mathrm{s}^{-1}$ or $0.3 \mathrm{~m} \cdot \mathrm{s}^{-1}$ are recommended because of practical applicability and body stabilization in asymptomatic patients. Clinicians are recommended to use more than one procedure to allow functional measurements based on the patient's abilities at the moment of the evaluation.

\section{References}

Andersen, L. L., Vinstrup, J., Jakobsen, M. D., \& Sundstrup, E. (2017). Validity and reliability of elastic resistance bands for measuring shoulder muscle strength. Scandinavian Journal of Medicine and Science in Sports, 27(8), 887-894. https://doi.org/10.1111/sms.12695

Andrade, M. D. S., Fleury, A. M., de Lira, C. A. B., Dubas, J. P., \& da Silva, A. C. (2010). Profile of isokinetic eccentric-to-concentric strength ratios of shoulder rotator muscles in elite female team handball players. Journal of Sports Sciences, 28(7), 743-749. https://doi.org/10.1080/02640411003645687

Beneka, A., Mailliou, P., Giannakopoulos, K., Kyrialanis, P., \& Godolias, G. (2002). Different training modes for the rotator cuff muscle group. A comparative study. Isokinetics and Exercise Science, 10(2), 73-79. Retrieved from http://cat.inist.fr/?aModele $=$ afficheN\&cpsidt $=13912456$

Broztman, S. B., \& Wilk, K. E. (2005). Rehabilitacion ortopedica clinica. Madrid, España: Elsevier.

Byram, I. R., Bushnell, B. D., Dugger, K., Charron, K., Harrell, F. E., \& Noonan, T. J. (2010). Preseason shoulder strength measurements in professional baseball pitchers: Identifying 
302

303

304

305

306

307

308

309

310

311

312

313

314

315

316

317

318

319

320

321

322

323

324

325

326

327

328

329

330

331

332

333

334

335

336

337

338

339

340

341

342

343

344

345

346

347

players at risk for injury. American Journal of Sports Medicine, 38(7), 1375-1382.

https://doi.org/10.1177/0363546509360404

Caruso, J. F., Brown, L. E., \& Tufano, J. J. (2012). The reproducibility of isokinetic dynamometry data. Isokinetics and Exercise Science, 20(4), 239-253. https://doi.org/10.3233/IES-2012-0477

Castro, M. P. de, Fonseca, P., Morais, S. T., Borgonovo-Santos, M., Coelho, E. F. C., Ribeiro, D. C., ... Morais, S. T. (2017). Functional shoulder ratios with high velocities of shoulder internal rotation are most sensitive to determine shoulder rotation torque imbalance: a crosssectional study with elite handball players and controls. Sports Biomechanics, 18(1), 39-50. https://doi.org/10.1080/14763141.2017.1380222

Cerda Vega, E., Jerez-Mayorga, D., Jara, C. C., Guzman-guzman, I., Ponce, A. R., \& Chirosa, L. J. (2018). Validity and reliability of evaluating hip abductor strength using different normalization methods in a functional electromechanical device. PLOS ONE, 8(13), 1-12.

Chamorro, C, Armijo-Olivo, S., De La Fuente, C., Fuentes, J., \& Chirosa, L. (2017). Absolute reliability and concurrent validity of hand held dynamometry and isokinetic dynamometry in the hip, knee and ankle joint: Systematic review and meta-analysis. Open Medicine (Poland), 12(1), 359-375. https://doi.org/10.1515/med-2017-0052

Chamorro, Claudio, De La Fuente, C., Jerez, D., Campos, C., \& Chirosa, L. J. (2018). Reliability of shoulder rotators isometric strength test using a novel pulley electromechanical dynamometer. Influence of the assessment position. Asian Journal of Sports Medicine, 9(2). https://doi.org/10.5812/asjsm.60406

Chamorro, Claudio, de la Fuente, C., Rubio, J., Campos, C., \& Chirosa, L. J. (2019). Absolute reliability and concurrent validity of a novel electromechanical pulley dynamometer for measuring shoulder rotation isometric strength in asymptomatic subjects. Study conducted at Pontificia Universidad Católica, Santiago, Chile. Journal of the Pakistan Medical Association, 69(7), 1000-1005.

Cohen, J. (1988). Statistical Power Analysis for the Behavioral Sciences (2nd ed.). New York: Lawrence Erlbaum Associates.

Cools, A. M., De Wilde, L., van Tongel, A., Ceyssens, C., Ryckewaert, R., \& Cambier, D. C. (2014). Measuring shoulder external and internal rotation strength and range of motion: Comprehensive intra-rater and inter-rater reliability study of several testing protocols. Journal of Shoulder and Elbow Surgery, 23(10), 1454-1461. https://doi.org/10.1016/j.jse.2014.01.006

Cools, A. M., Johansson, F. R., Borms, D., \& Maenhout, A. (2015). Prevention of shoulder injuries in overhead athletes: A science-based approach. Brazilian Journal of Physical Therapy, 19(5), 331-339. https://doi.org/10.1590/bjpt-rbf.2014.0109

Courel-Ibáñez, J., Martínez-Cava, A., Morán-Navarro, R., Escribano-Peñas, P., ChavarrenCabrero, J., González-Badillo, J. J., \& Pallarés, J. G. (2019). Reproducibility and Repeatability of Five Different Technologies for Bar Velocity Measurement in Resistance Training. Annals of Biomedical Engineering. https://doi.org/10.1007/s10439-019-02265-6

Dvir, Z., \& Müller, S. (2019). Multiple-Joint Isokinetic Dynamometry: A Critical Review. Journal of Strenght and Conditioning Research, 00(00), 1-15.

Edouard, P., Codine, P., Samozino, P., Bernard, P. L., Hérisson, C., \& Gremeaux, V. (2013). Reliability of shoulder rotators isokinetic strength imbalance measured using the Biodex dynamometer. Journal of Science and Medicine in Sport, 16(2), 162-165. https://doi.org/10.1016/j.jsams.2012.01.007 
348

349

350

351

352

353

354

355

356

357

358

359

360

361

362

363

364

365

366

367

368

369

370

371

372

373

374

375

376

377

378

379

380

381

382

383

384

385

386

387

388

389

390

391

392

393

Edouard, P., Samozino, P., Julia, M., Gleizes Cervera, S., Vanbiervliet, W., Calmels, P., \& Gremeaux, V. (2011). Reliability of isokinetic assessment of shoulder-rotator strength: a systematic review of the effect of position. Journal of Sport Rehabilitation, 20(3), 367-383. https://doi.org/10.1123/jsr.20.3.367

Fàbrica, C. G., Ferraro, D., Mercado-Palomino, E., Molina-Molina, A., \& Chirosa-Rios, I. (2020). Differences in Utilization of Lower Limb Muscle Power in Squat Jump With Positive and Negative Load. Frontiers in Physiology, 11(June), 1-8. https://doi.org/10.3389/fphys.2020.00573

Forthomme, B., Dvir, Z., Crielaard, J. M., \& Croisier, J. L. (2011). Isokinetic assessment of the shoulder rotators: A study of optimal test position. Clinical Physiology and Functional Imaging, 31(3), 227-232. https://doi.org/10.1111/j.1475-097X.2010.01005.X

Frisiello, S., Gazaille, A., O’Halloran, J., Palmer, M. L., \& Waugh, D. (1994). Test-Retest Reliability of Eccentric Peak Torque Values for Shoulder Medial and Lateral Rotation Using the Biodex Isokinetic Dynamometer. Journal of Orthopaedic and Sports Therapy, 19(6), 341-344.

García, J. A. (2006). Entrenamiento en Balonmano: Bases de la... Paidotribo. https://doi.org/10.7707/hmj.v5i3.191

Greenfield, B. H., Donatelli, R., Wooden, M. J., \& Wilkes, J. (1990). Isokinetic evaluation of shoulder rotational strength between the plane of scapula and the frontal plane. / Evaluation isocinetique de la force rotatoire de l'epaule entre le plan de l'omoplate et le plan frontal. American Journal of Sports Medicine, 18(2), 124-128. Retrieved from http://articles.sirc.ca/search.cfm?id=254553\%5Cnhttp://ezproxy.library.uvic.ca/login?url=ht tp://search.ebscohost.com/login.aspx?direct=true \&db=sph\&AN=SPH254553\&site=ehostlive\&scope $=$ site $\% 5 \mathrm{Cnhttp}: / /$ www.aossm.org/

Hadzic, V., Ursej, E., Kalc, M., \& Dervisevic, E. (2012). Reproducibility of shoulder short range of motion isokinetic and isometric strength testing. Journal of Exercise Science and Fitness, 10(2), 83-89. https://doi.org/10.1016/j.jesf.2012.10.005

Holt, K. L., Raper, D. P., Boettcher, C. E., Waddington, G. S., \& Drew, M. K. (2016). Hand-held dynamometry strength measures for internal and external rotation demonstrate superior reliability, lower minimal detectable change and higher correlation to isokinetic dynamometry than externally-fixed dynamometry of the shoulder. Physical Therapy in Sport, 21, 75-81. https://doi.org/10.1016/j.ptsp.2016.07.001

Hopkins, W. G. (2000). Mesaures of reliability in sports medicine and science. Sports Medicine, $30(1), 1-15$.

Hopkins, W. G., Marshall, S. W., Batterham, A. M., \& Hanin, J. (2009). Progressive statistics for studies in sports medicine and exercise science. Medicine and Science in Sports and Exercise, 41(1), 3-12. https://doi.org/10.1249/MSS.0b013e31818cb278

Hopkins, W., Schabort, E., \& Hawley, J. (2001). Reliability of power in physical performance tests. Sports Medicine, 31(3), 211-234. https://doi.org/10.2165/00007256-20013103000005

Johansson, F. R., Skillgate, E., Lapauw, M. L., Clijmans, D., Deneulin, V. P., Palmans, T., ... Cools, A. M. (2015). Measuring eccentric strength of the shoulder external rotators using a handheld dynamometer: Reliability and validity. Journal of Athletic Training, 50(7), 719725. https://doi.org/10.4085/1062-6050-49.3.72

Kramer, J. F., \& Ng, L. R. (1996). Static and Dynamic Strength of the Shoulder Rotators in Healthy, 45- to 75-Year-Old Men and Women. Journal of Orthopaedic \& Sports Physical 
394

395

396

397

398

399

400

401

402

403

404

405

406

407

408

409

410

411

412

413

414

415

416

417

418

419

420

421

422

423

424

425

426

427

428

429

430

431

432

433

434

435

436

437

438

439
Therapy, 24(1), 11-18. https://doi.org/10.2519/jospt.1996.24.1.11

Lu, T., Hsu, H., Chang, L., \& Chen, H. (2007). Enhancing the examiner's resisting force improves the reliability of manual muscle strength measurements: comparison of a new device with hand-held dynamometry. Journal of Rehabilitation Medicine, (39), 679-684.

Malerba, J. L., Adam, M. L., Harris, B. A., \& Krebs, D. E. (1993). Reliability of Dynamic and Isometric Testing of Shoulder External and Internal Rotators. Journal of Orthopaedic \& Sports Physical Therapy, 18(4), 543-552. https://doi.org/10.2519/jospt.1993.18.4.543

Mayer, F., Horstmann, T., Kranenberg, U., Röcker, K., \& Dickhuth, H.-H. (1994). Reproducibility of Isokinetic Peak Torque and Angle at Peak Torque in the Shoulder Joint. International Journal of Sports Medicine, 15(S 1), S26-S31. https://doi.org/10.1055/s2007-1021106

McLaine, S. J., Ginn, K. A., Kitic, C. M., Fell, J. W., \& Bird, M.-L. (2016). The Reliability of Strength Tests Performed In Elevated Shoulder Positions Using a Handheld Dynamometer. Journal of Sport Rehabilitation, 25(2), 1-4. https://doi.org/10.1123/jsr.2015-0034

Nugent, E. P., Snodgrass, S. J., \& Callister, R. (2015). The effect of velocity and familiarisation on the reproducibility of isokinetic dynamometry. Isokinetics and Exercise Science, 23(3), 205-214. https://doi.org/10.3233/IES-150582

Paalanne, N. P., Korpelainen, R., Taimela, S. P., Remes, J., Salakka, M., \& Karppinen, J. I. (2009). Reproducibility and reference values of inclinometric balance and isometric trunk muscle strength measurements in finnish young adults. Journal of Strength and Conditioning Research, 23(5), 1618-1626. https://doi.org/10.1519/JSC.0b013e3181a3cdfc

Pallarés, J. G., Sánchez-Medina, L., Pérez, C. E., De La Cruz-Sánchez, E., \& Mora-Rodriguez, R. (2014). Imposing a pause between the eccentric and concentric phases increases the reliability of isoinertial strength assessments. Journal of Sports Sciences, 32(12), 11651175. https://doi.org/10.1080/02640414.2014.889844

Radaelli, R., Bottaro, M., Weber, F., Brown, L. E., \& Pinto, R. S. (2010). Influence of body position on shoulder rotator muscle strength during isokinetic assessment. Isokinetics and Exercise Science. https://doi.org/10.3233/IES-2010-0369

Rodriguez-Perea, A., Ríos, L. J. C., Martinez-Garcia, D., Ulloa-Díaz, D., Rojas, F. G., JerezMayorga, D., \& Rios, I. J. C. (2019). Reliability of isometric and isokinetic trunk flexor strength using a functional electromechanical dynamometer. PeerJ, 2019(10), 1-17. https://doi.org/10.7717/peerj.7883

Rodríguez-Rosell, D., Pareja-Blanco, F., Aagaard, P., \& González-Badillo, J. J. (2017). Physiological and methodological aspects of rate of force development assessment in human skeletal muscle. Clinical Physiology and Functional Imaging, 1-20. https://doi.org/10.1111/cpf.12495

Stickley, C. D., Hetzler, R. K., Freemyer, B. G., \& Kimura, I. F. (2008). Isokinetic Peak Torque Ratios and Shoulder Injury History in Adolescent Female Volleyball Athletes. Journal of Athletic Training, 43(6), 571-577.

Wagner, H., Pfusterschmied, J., Tilp, M., Landlinger, J., von Duvillard, S. P., \& Müller, E. (2012). Upper-body kinematics in team-handball throw, tennis serve, and volleyball spike. Scandinavian Journal of Medicine and Science in Sports, 24(2), 345-354. https://doi.org/10.1111/j.1600-0838.2012.01503.x

Weir, J. P. (2005). Quantifying test-retest reliability using the intraclass correlation coefficient and the SEM. Journal of Strength and Conditioning Research, 19(1), 231-240. https://doi.org/10.1519/15184.1 
440 Zanca, G. G., Oliveira, A. B., Saccol, M. F., \& Mattiello-Rosa, S. M. (2011). Isokinetic 441 dynamometry applied to shoulder rotators - Velocity limitations in eccentric evaluations.

$442 \quad$ Journal of Science and Medicine in Sport, 14(6), 541-546.

$443 \quad$ https://doi.org/10.1016/j.jsams.2011.05.002

444

445

446

447 Table 1. Reliability of the shoulder strength obtained during the isokinetic test on FEMD. Data 448 are expressed in mean \pm SD in Newtons $(\mathrm{N})$, ICC, CV and SEM of the dominant arm strength 449 obtained for two evaluation sessions

450

451

452

453

454

455 Figure 3. Subjection system of own manufacture.

456

457 Figure 4. Bland-Altman plots of test-retest for internal and internal rotation at $0,3 \mathrm{~m} \cdot \mathrm{s}^{-1}$ and 0,6 $458 \mathrm{~m} \cdot \mathrm{s}-1$.

459

460

Figure 5. Bland-Altman plots of test-retest for internal and external rotation at $0,3 \mathrm{~m} \cdot \mathrm{s}^{-1}$ and 0,6 $461 \mathrm{~m} \cdot \mathrm{s}-1$.

462 


\section{Table $\mathbf{1}$ (on next page)}

Reliability of the shoulder strength obtained during the isokinetic test on Dynasystem dynamometer

SD: Standard Deviation, ES: Effect Size, ICC: Intraclass Correlation Coefficient, Cl: Confidence Intervals, CV: Coefficient of Variation, SEM: Standard Error of Measurement 
Tablle 1. Reliability of the shoulder strength obtained during the isokinetic test on Dynasystem dynamometer

\begin{tabular}{|c|c|c|c|c|c|c|c|c|c|}
\hline & \multirow{2}{*}{\multicolumn{2}{|c|}{ Parameters }} & \multicolumn{2}{|c|}{ Mean \pm SD } & \multirow{2}{*}{ ES } & \multirow{2}{*}{ ICC } & \multirow{2}{*}{$\begin{array}{c}95 \% \mathrm{Cl} \\
\text { lower-upper }\end{array}$} & \multirow{2}{*}{ CV (\%) } & \multirow{2}{*}{ SEM (\%) } \\
\hline & & & Session 1 & Session 2 & & & & & \\
\hline \multirow{4}{*}{$\begin{array}{l}\text { Internal } \\
\text { Rotation }\end{array}$} & \multirow{2}{*}{$0,3 \mathrm{~m} \cdot \mathrm{s}^{-1}$} & Concentric & $83,10 \pm 17.6$ & $81,40 \pm 16,1$ & $-0,10$ & 0,85 & $0,71-0,92$ & 8,27 & 6,8 \\
\hline & & Eccentric & $206,50 \pm 34,9$ & $201,20 \pm 31,8$ & $-0,16$ & 0,81 & $0,64-0,90$ & 7,38 & 15,04 \\
\hline & \multirow{2}{*}{$0,6 \mathrm{~m} \cdot \mathrm{s}^{-1}$} & Concentric & $88,80 \pm 20,6$ & $88,10 \pm 19,6$ & $-0,03$ & 0,93 & $0,86-0,96$ & 6,31 & 5,58 \\
\hline & & Eccentric & $218,00 \pm 39,3$ & $218,20 \pm 41,6$ & 0,00 & 0,87 & $0,75-0,93$ & 6,87 & 14,98 \\
\hline \multirow{4}{*}{$\begin{array}{l}\text { External } \\
\text { Rotation }\end{array}$} & \multirow{2}{*}{$0,3 \mathrm{~m} \cdot \mathrm{s}^{-1}$} & Concentric & $64,00 \pm 13,2$ & $67,00 \pm 11,9$ & 0,24 & 0,90 & $0,80-0,95$ & 6,39 & 4,19 \\
\hline & & Eccentric & $198,90 \pm 89$ & $196,00 \pm 89,9$ & $-0,03$ & 0,98 & $0,96-0,99$ & 6,91 & 13,64 \\
\hline & \multirow{2}{*}{$0,6 \mathrm{~m} \cdot \mathrm{s}^{-1}$} & Concentric & $64,40 \pm 11,5$ & $65,50 \pm 12,1$ & 0,10 & 0,89 & $0,78-0,94$ & 6,26 & 4,06 \\
\hline & & Eccentric & $201,70 \pm 32,6$ & $194,90 \pm 38,1$ & $-0,19$ & 0,92 & $0,85-0,96$ & 5,12 & 10,16 \\
\hline
\end{tabular}

\section{2}

3

4 
Figure 1

FEMD Dynasystem.

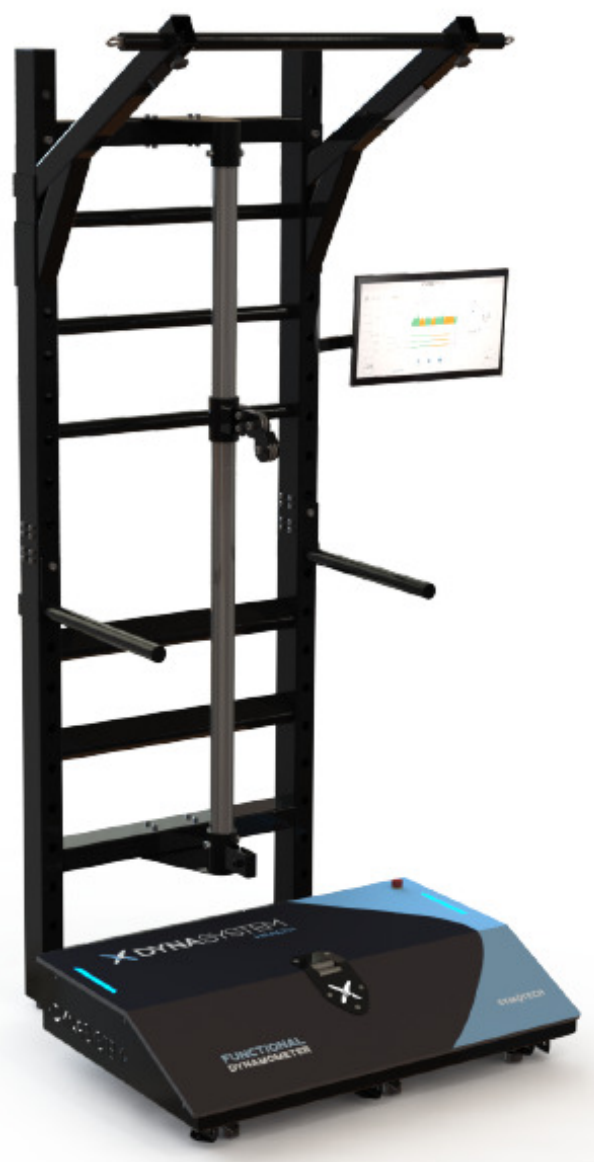


Figure 2

FEMD data screen

A Tónico

Series

\section{$1 / 1$}

Repeticiones 6

Rocorrido (cm)

20

Concesntrico (Kg)
\[ 8.0 \text { a } 8.0 \]

Excentrico $(\mathrm{kg})$ a 8.0

Inc. Reperticion (Kg) 2.0

\section{$0: 07$}

Fuerza

Fuerza - Tiempo (Kg-s)
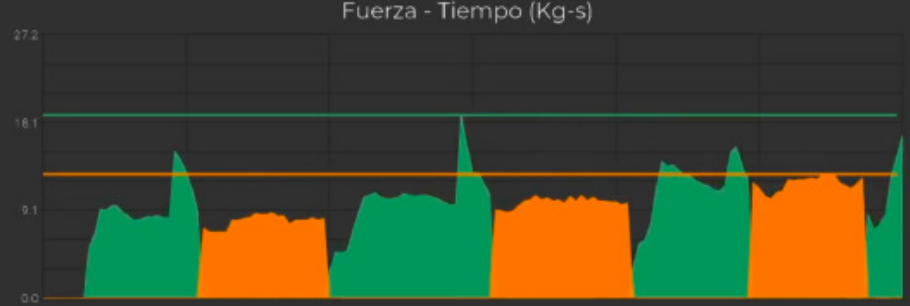

Fuerza media (Kg)

11.8

ค $16.8 \%$

^ $19.4 \%$

11.7

\section{8}

0.0

w 


\section{Figure 3}

Subjection system of own manufacture

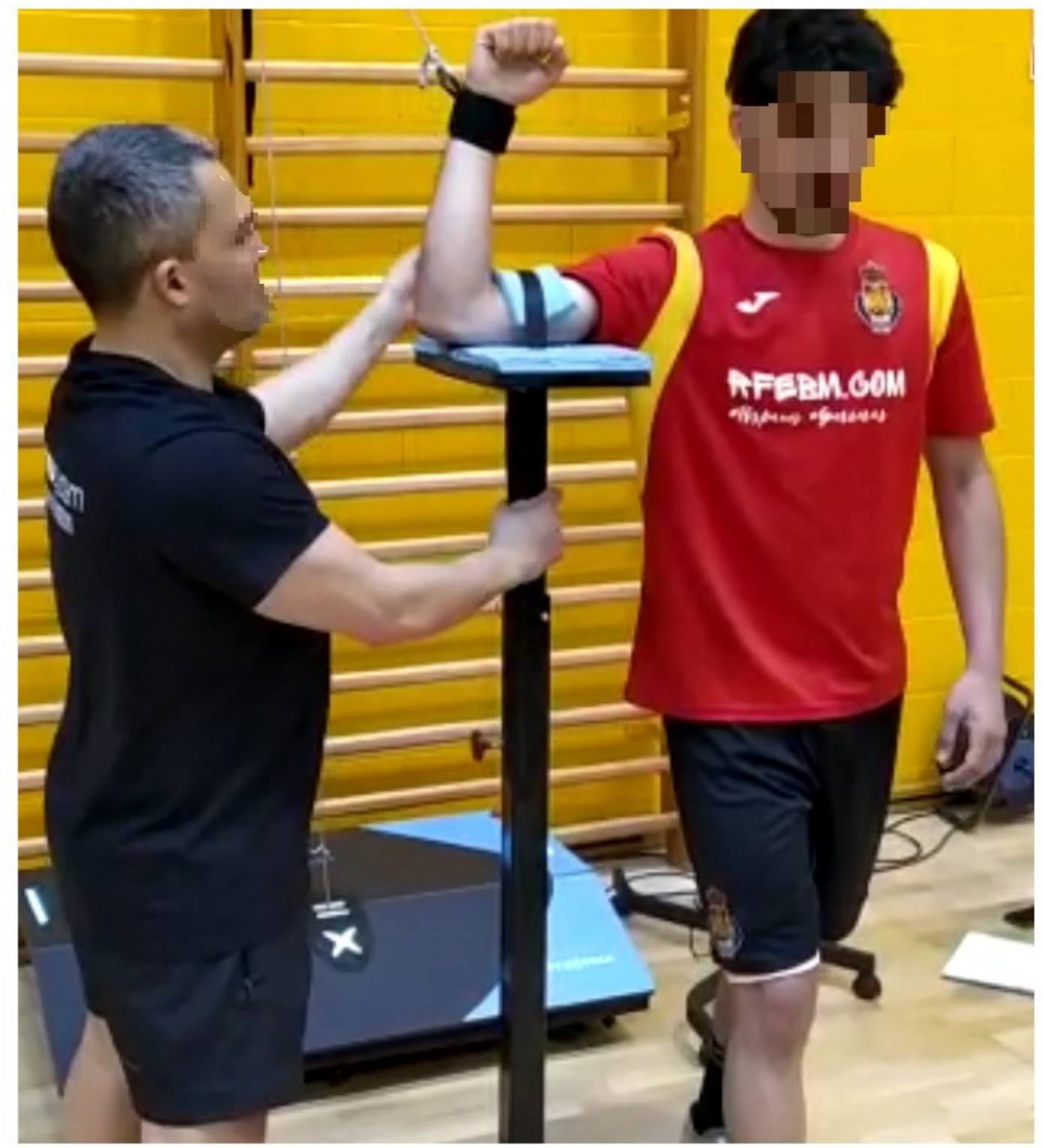


Figure 4

Bland-Altman plots of test-retest for internal and internal rotation at $0,3 \mathrm{~m} \cdot \mathrm{s}^{-1}$ and 0,6 $\mathrm{m} \cdot \mathrm{s}-1$.
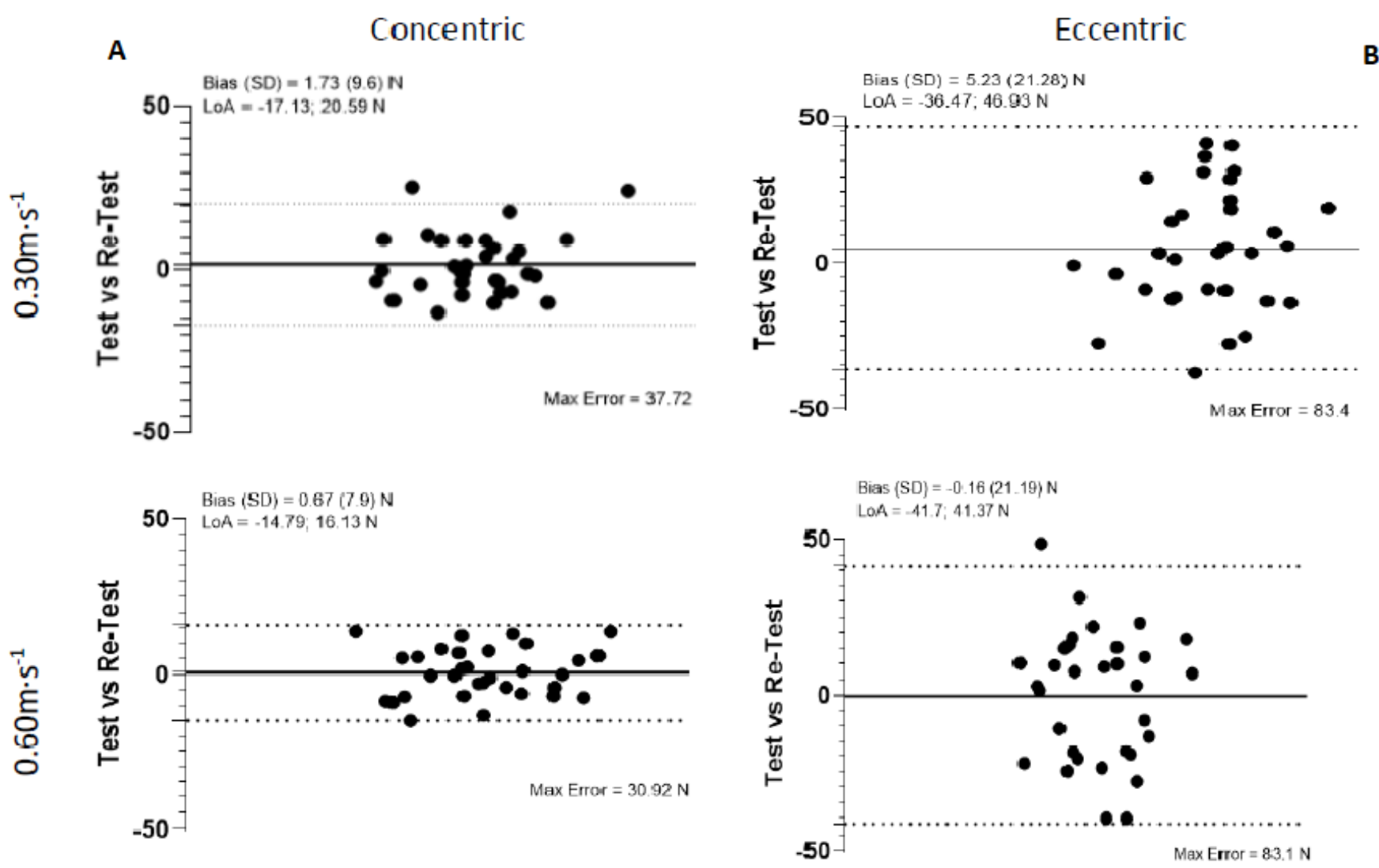

C

D 
Figure 5

Bland-Altman plots of test-retest for internal and external rotation at $0,3 \mathrm{~m} \cdot \mathrm{s}^{-1}$ and 0,6 $m \cdot s-1$.

Concentric

A
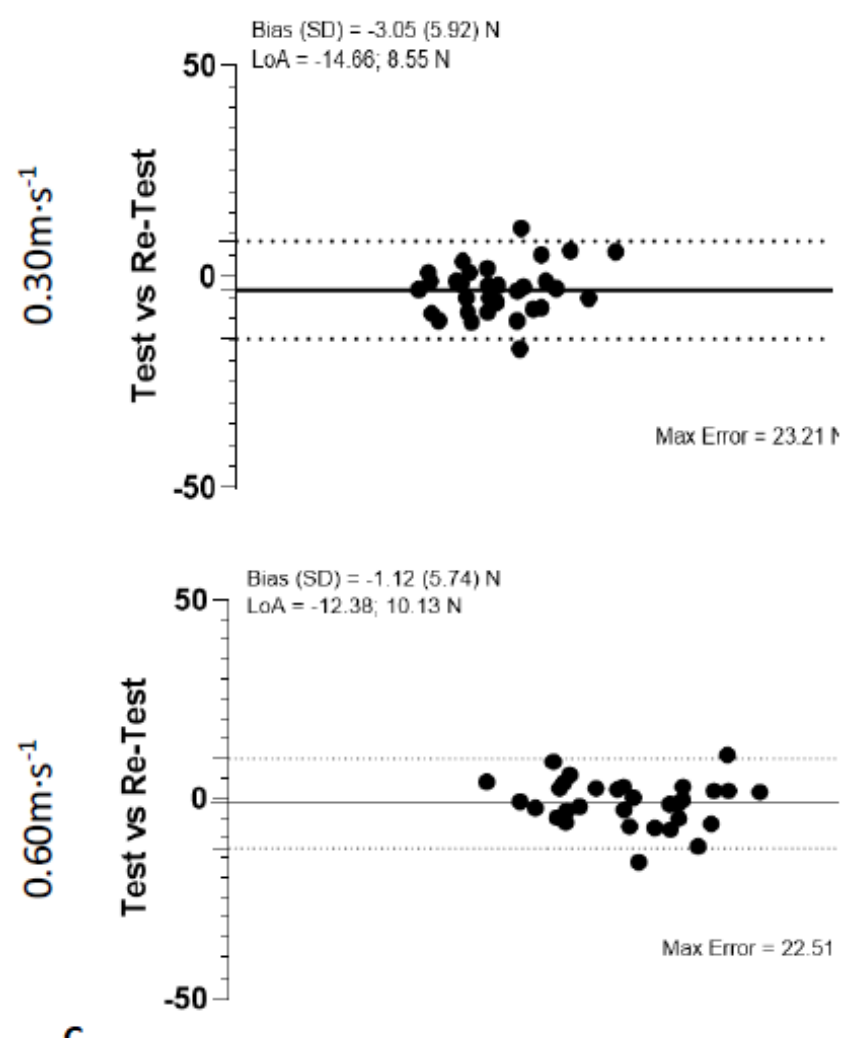

Eccentric
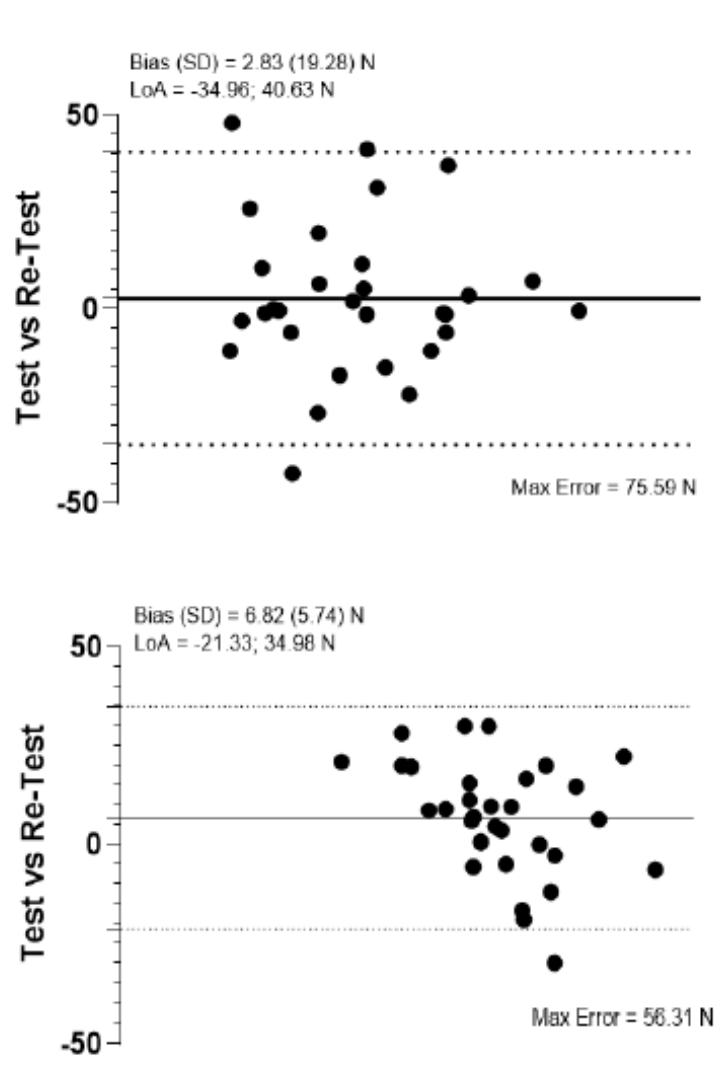